\title{
Extraction and antibacterial evaluation of marine AMPs against diabetic wound pathogens
}

\author{
Deepak Tom Jose ${ }^{1}$, C. Uma ${ }^{1}$, P. Sivagurunathan ${ }^{1}$, B. Aswini ${ }^{1}$, M. D. Dinesh ${ }^{2}$ \\ ${ }^{1}$ Department of Microbiology, Annamalai University, Chidambaram, India. \\ ${ }^{2}$ Department of Microbiology, Pazhassiraja College, Pulpally, Kerala.
}

\section{ARTICLE INFO \\ Received on: 04/05/2020 \\ Accepted on: 19/08/2020 \\ Available online: 05/11/2020}

Key words:

Antimicrobial peptides, diabetic foot ulcer, pyogenic

bacteria, wound healing,

biocompatibility.

\begin{abstract}
The need for an alternative wound dressing material with effective coatings using antimicrobial peptides (AMPs) from a marine fish (Carangoides malabaricus) was selected as the primary objective in the present research. The AMPs were extracted and purified using standard biochemical methods and the fractional concentrations were selected to coat nonadherent wound dressing materials. Antibacterial activity, drug release behavior, and biocompatibility of the developed dressing material were studied. As the antibacterial activity, drug releasing behavior, and biocompatible properties of the coated materials showed promising results, a novel tissue-engineered wound dressing material can be developed to meet the challenges in the medical industry in the near future. This application would act as a skin replacement or an equivalent material to treat diabetic foot ulcer cases caused by pyogenic bacterial species.
\end{abstract}

\section{INTRODUCTION}

Wound is defined as the disruption or damage of the functions of skin cells associated with underlying tissues. It may even extend to different organs internally or externally (Sabine et al., 2014). Wounds get contaminated by different types of pyogenic bacteria which may or may not be treated with antibiotics. The reason is not described clearly elsewhere, except in diabetic cases. The use of antibiotics in diabetic wound cases is reported to cause different types of multi-drug-resistant bacteria like Pseudomonas aeruginosa, Methicillin-resistant Staphylococcous aureus, Vancomycin-resistant Lactobacilli, Streptococci, Staphylococcus epidermidis, Acinetobacter sp., Bacteriodes fragilis, and Peptostreptococcus sp. (Elshafei et al., 2011).

Wound healing is a natural process that depends on a series of overlapping events influenced by intrinsic and extrinsic factors and the growth and proliferation of fibroblast cells at the wound site. The precursor cells need to be stimulated for

\section{"Corresponding Author}

C. Uma, Department of Microbiology, Annamalai University, Chidambaram,Tamil Nadu.E-mail:umasaravanan1@gmail.com fibroblast proliferation (Boateng et al., 2008). Simultaneously, cell proliferation and reduction in bacterial numbers and tissue repair are essential for wound healing at the target site. The target wound site in diabetic cases was mainly reported on the foot (diabetic foot ulcers). Improper therapeutic drugs may lead to unconditional treatment of diabetic foot ulcer cases with improper fibroblast cell proliferation and increased physiological actions of pyogenic bacterial species also known as critical colonization at the open wound site (White, 2002).

Many research works were conducted to overcome this situation worldwide by developing and fabricating antimicrobial wound dressing materials. These novel materials are commercialized and available in medical markets as degradable hydrogels, nonadherent polyesters, biodegradable polyvinyl alcohol (PVA)-based polymers, nanometal-coated bandages, antibiotic impregnated mesh, and hydrocolloids (Elbadawy et al., 2017). Among these types of materials, if an appropriate wound dressing is not selected, then it may lead to systemic cytotoxicity, critical colonization of drugresistant organisms (Flores and Kingsley, 2007), and need for amputation. Hence, the need for the development of new antimicrobials from a natural source is considered significant in the present research. 
As previously reported on the medically significant peptides from different sources by many researchers, marine antimicrobial peptides (AMPs) were selected in the present study for the development of a novel antimicrobial dressing material.

Marine fishes rely on the innate immune system to combat against the infection-causing pathogens, which is the first-line natural defense mechanism. The immune system of marine species depends on the synthesis of AMPs natively in their biological ecosystem (Rameshkumar et al., 2009). The researchers proved that the AMPs of marine species would be synthesized in their hemolymph. Reports are already published on the way of synthesizing AMPs at low cost, storage conditions, maintaining sterility, and availability for medical applications (Michel et al., 2019).

Based on the need for an alternative wound dressing material with effective antibacterial coatings to provide significant therapy against diabetic wound pathogens and also to avoid antibiotic-resistant characters among them, AMPs from one such marine fish (Carangoides malabaricus) was studied in the present research work. The AMPs were extracted and purified using standard biochemical methods and the fractional concentrations were selected to coat nonadherent wound dressing materials. Antibacterial activity, drug release behavior, and biocompatibility of the developed dressing material were studied. This approach would pave the way for developing a novel tissue-engineered wound dressing material in the future; the success of the product would even act as a skin replacement to meet the biomedical challenges.

\section{MATERIALS AND METHODS}

Purchase of wound healing mesh materials and marine fish (Carangoides praeustus)

The present research work was carried out in the Department of Microbiology, Annamalai University, Chidambaram, Tamil Nadu, India. The work was done during the period of November 2019 to February 2020. C. malabaricus (paarai) fish was purchased from the local fish market at Chidambaram, Tamil Nadu, India. Nonadhering wound dressing material was used in the study. In Table 1, the sample material designation and the product description are mentioned. PVA used as carrier was purchased from chemical suppliers (Hi Media, India)

\section{Extraction of AMPs from $C$. praeustus}

Carangoides malabaricus fish procured from the fish market was processed for the extraction of AMPs using the procedure described by Nieto Lozano et al. (2012) (Fig. 1). The fishes were cut into pieces and the tissues were collected in a sterile container. About $100 \mathrm{~g}$ of tissues were mixed with $10 \%$ acetic acid and homogenized for 1 hour in a homogenizer. The homogenized tissue mixtures were centrifuged at 10,000 rpm for 20 minutes at $4^{\circ} \mathrm{C}$. The supernatants were collected and equal volumes of acetone was mixed and centrifuged at 5,000 rpm for 10 minutes at $4^{\circ} \mathrm{C}$. Supernatant containing AMPs were purified using standard ammonium sulfate precipitation and dialysis process. AMPs as protein precipitates were collected finally and stored at $4^{\circ} \mathrm{C}$.

\section{Antibacterial activity of AMPs}

To determine the effective concentration of AMPs for coating the wound dressing mesh materials, antibacterial activity of four different concentrations (1X-10, 2X-20, 3X-30 and 4X$40 \mu \mathrm{g} / \mathrm{ml}$ ) were tested against the test organisms (Escherichia coli, Klebsiella pneumoniae, Acinetobacter baumannii, S. aureus, and P. aeruginosa) (Sasmita et al., 2018).

Selection of AMP concentrations based on the antibacterial activity was carried out using modified Kirby-Bauer test method - Agar well diffusion method. Briefly, sterile MullerHinton agar plates were prepared. The plates were allowed to solidify for 5 minutes and $0.1-\mathrm{ml}$ inoculum suspension of test bacteria were swabbed uniformly on the agar surface separately. After swabbing the cultures, four 6-mm wells were punctured on the agar surface using a sterile well borer for the addition of four different concentrations. All the plates after inoculation and addition of AMP concentrations were incubated at $37^{\circ} \mathrm{C}$ for 24 hours. The antibacterial activity of each concentration against the test bacteria was evaluated based on the inhibitory zones around the well. Experiments were carried out in triplicates and antibacterial activity was expressed in standard deviation values using the Statistical Package for Social Sciences (SPSS 9 for Windows 7.0) software.

\section{Coating wound dressing materials with AMPs + PVA}

Antibacterial coating of wound healing mesh material using the AMPs was done by a standard two dip-coating

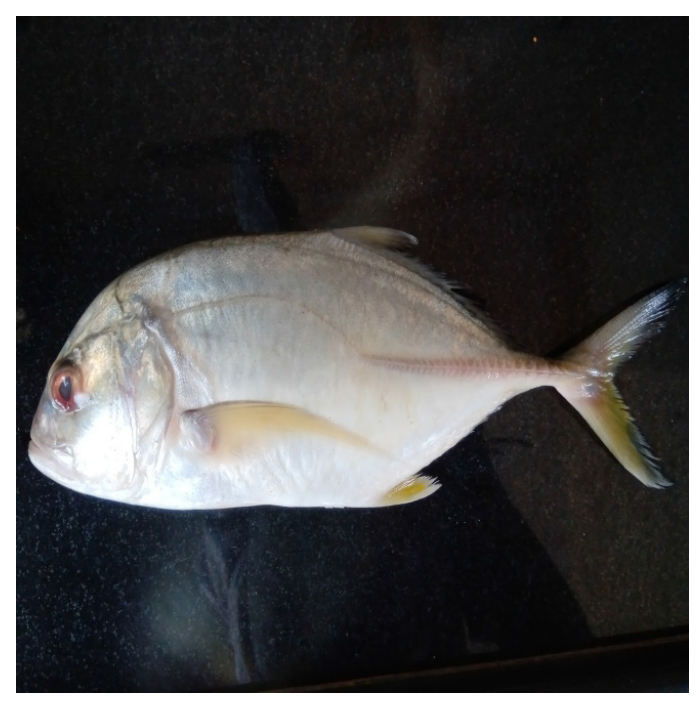

Figure 1. Selection of C. malabaricus fish for extraction of AMP.

Table 1. Details of wound dressing material.

\begin{tabular}{cll}
\hline S. No. & Product designation & Product description \\
\hline 1 & Polyester + TLC & Polyester nonadhering wound dressing mesh with thin lipido-colloid (TLC) matrix \\
\hline
\end{tabular}


technique (Gollwitzer et al., 2003). The technique started with the preparation of stable slurry with a specific amount $(5 \mathrm{~g})$ of AMPs in the molten polyethylene glycol (PEG). PEG (5 g) with a predefined molecular weight was mixed with AMPs $(10 \mathrm{ml}$ of purified fractions of AMPs) in a glass vial. The mixture was heated at the range of $60^{\circ} \mathrm{C}-70^{\circ} \mathrm{C}$ in a water bath to obtain homogeneous slurry. The resulting slurry was homogenized in a magnetic stirrer for 5-10 minutes. The wound healing mesh disk was cut $(10 \mathrm{~mm})$ using sterile devices and dip-coated twice with intermittent drying (suspension coating method).

The dip-coating procedure was carried out in sterile glass beakers on a shaker (120 rpm) for 30 minutes, with a drying period of about 15 minutes between the two coating procedures, followed by drying at room temperature. All the coating steps were carried out under strict aseptic conditions. After the coating procedure, the materials were stored at $4^{\circ} \mathrm{C}$ for up to 15 minutes. In order to increase antimicrobial drug loading and prevent excessive increase in material thickness, the coating process were repeated for replicates of each sample. Subsequently, in order to slow down the release rate of antimicrobial drug from PEG coating and mitigate the friction effect between material surfaces, a second coating layer was formed using PVA. PVA (drug carrier) was dissolved in DMSO to acquire a $10 \%(w / w)$ solution. PEGcoated materials were submerged into PVA solution three times for 1 minute each. The coated materials were left to dry on a clean bench for 24-48 hours at room temperature to remove residual DMSO, followed by determining the antimicrobial activity.

\section{Qualitative antibacterial activity of AMPs + PVA-coated materials}

AMP-coated wound dressing materials were subjected to evaluate the qualitative antibacterial activity against the test organisms individually (El-Rehewy et al., 2009). The standard disk diffusion method was used in the research to evaluate the antibacterial activity. Briefly, Mueller-Hinton agar (MHA) plates were prepared by pouring $15 \mathrm{ml}$ of media into sterile Petri dishes. The plates were allowed to solidify for 5 minutes and 0.1 $\mathrm{ml}$ inoculum was swabbed uniformly and allowed to dry for 60 seconds. Premeasured disk-shaped $(20 \mathrm{~mm}$ in diameter) AMPcoated wound dressing materials were placed on the MHA plates (seeded with bacterial inoculum). The disk was gently pressed to attach on the agar surface using sterile conditions. A plain mesh without AMPs was also kept in the plate as control. All the plates were incubated at $37^{\circ} \mathrm{C}$ for $24-48$ hours. At the end of incubation, the zone of inhibition formed around each material was measured in millimeter. Experiments were carried out in triplicates and antibacterial activity was expressed in standard deviation values using SPSS 9 (for Windows 7.0) software.

\section{Determining the release concentration of AMPs from coated materials}

The release of AMPs from the coated wound dress materials was studied using a standard in vitro drug release analysis (modified method of Mashru and Saikumar, 2010). The sample material was coated using the method described in previous section (Gollwitzer et al., 2003). Drug release was studied in 100-ml phosphate buffer ( $\mathrm{pH}$ 6.8). The apparatus setup was made using a 250-ml glass beaker placed on a magnetic stirrer. A magnetic bead washed well was used for the experiment. The stirring condition was kept constant at $150 \mathrm{rpm}$. The release profiles were evaluated spectrophotometrically using UV-VIS spectrophotometer. Absorbance was measured for $4 \mathrm{ml}$ of the sample solution at 450 $\mathrm{nm}$ wave length against phosphate-buffered saline as a blank. A fresh medium of the same volume was replaced each time after measuring absorbance. The drug release profile of coated wound dressing material was studied during the time period $(0.5,1,2,4$, $8,12,24,48,72,96$, and 120 hours) using a standard calibration curve.

\section{Assessing the biocompatibility of AMPs + PVA-coated materials}

3-(4,5-Dimethylthiazol-2-yl)-2,5-diphenyltetrazolium bromide (MTT) assay was used to assess the biocompatibility of AMPs + PVA-coated wound dressing materials (Budman et al., 2012). $\mathrm{L}_{929}$ fibroblast cell lines were cultivated in 12 -well microtitre plates to reach a confluent cell growth. The samples were applied directly to the developed fibroblast monolayer. Before cell seeding, the specimens were pre-wetted in $70 \%$ aqueous ethanol solution for 48 hours; rinsed twice with ultrapure water, and immersed in 1-ml DMEM fibroblast medium in 24-well plates for 2 hours in an incubator at $37^{\circ} \mathrm{C}$. The specimens were then seeded with $\mathrm{L}_{929}$ fibroblast cell line at 10,000 cells per well according to the routine cell-culture methods. The plates were incubated at $37^{\circ} \mathrm{C}$ and $5 \%$ $\mathrm{CO}_{2}$ for 15 days. The effect of AMPs on fibroblast viability was evaluated using the photometric MTT assay. At each time point, samples were taken from the 24-well plates and transferred into new plates for the MTT study. About $1 \mathrm{ml}$ of MTT solution was added to each well and the plates were incubated for 3 hours. All the wells were rinsed and desorbed in $100 \mu \mathrm{l}$ of $70 \%$ isopropanol for the formation of purple crystals. The plates were agitated rapidly at $400 \mathrm{rpm}$ for 40 minutes and the purple crystal dyed solution was transferred to a 96 -well microtitre plate and read at $550 \mathrm{~nm}$. The viability is expressed as a percentage of the control sample (100\%).

\section{RESULTS AND DISCUSSION}

\section{Antibacterial activity of AMPs}

To determine the effective AMP concentration for coating the wound dressing mesh materials, antibacterial activity of four different concentrations were tested against the test organisms. During the analysis, the higher concentrations (4X strength $-40 \mu \mathrm{g} / \mathrm{ml}$ ) expressed more inhibitory zones against all test organisms than the other lower concentrations. $1 \mathrm{X}$ concentration did not inhibit any of the test organisms during the analysis. In Figure 2, the difference in size of inhibitory zones among all the concentrations was clearly presented. In Table 2, the inhibitory zones measured in millimeter also supported the above results in the images. Among the test organisms, E. coli and K. pneumoniae exhibited inhibitory zones of $12.6 \pm 1.08,16.2 \pm 1.08$, and 22.6 $\pm 1.04 \mathrm{~mm}$ and $10.2 \pm 0.57,12.6 \pm 1.04$, and $18.3 \pm 0.86 \mathrm{~mm}$ for 2X, 3X, and 4X concentrates, respectively. Enterobacter sp. and $S$. aureus exhibited inhibitory zones of $11.1 \pm 1.04,12.5 \pm 0.86$, and $19.2 \pm 0.76 \mathrm{~mm}$ and $10.1 \pm 1.25,11.6 \pm 0.29$, and $20.1 \pm$ 

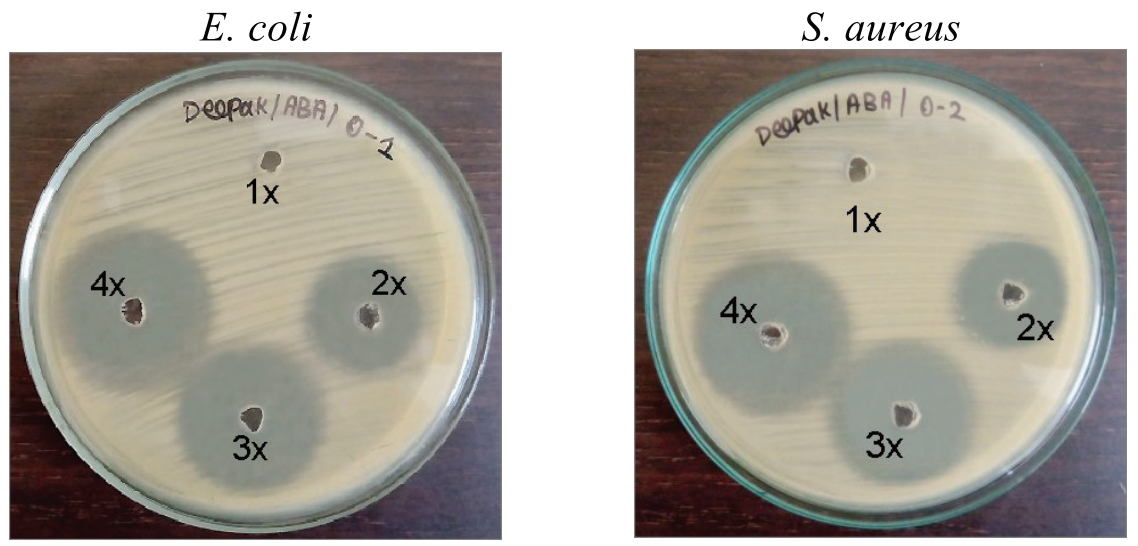

Figure 2. Antibacterial activity of AMPs. $1 X-10 \mu \mathrm{g} / \mathrm{ml}, 2 X-20 \mu \mathrm{g} / \mathrm{ml}, 3 X-30 \mu \mathrm{g} / \mathrm{ml}$, and $4 X-40 \mu \mathrm{g} / \mathrm{ml}$.

Table 2. Selection of AMP concentration based on the antibacterial activity of AMPs. $<$

\begin{tabular}{clcccc}
\hline \multirow{2}{*}{ S. No } & Test organism & \multicolumn{4}{c}{ Zone of inhibition (mm) } \\
\cline { 3 - 6 } & & $\mathbf{1 X}$ & $\mathbf{2 X}$ & $\mathbf{3 X}$ & $\mathbf{4 X}$ \\
\hline 1 & E. coli & 0 & $12.6 \pm 1.08$ & $16.2 \pm 1.08$ & $22.6 \pm 1.04$ \\
2 & K. pneumoniae & 0 & $10.2 \pm 0.57$ & $12.6 \pm 1.04$ & $18.3 \pm 0.86$ \\
3 & Enterobacter $\mathrm{sp}$ & 0 & $11.1 \pm 1.04$ & $12.5 \pm 0.86$ & $19.2 \pm 0.76$ \\
4 & S. aureus & 0 & $10.1 \pm 1.25$ & $11.6 \pm 0.29$ & $20.1 \pm 1.6$ \\
5 & S. epidermidis & 0 & $11.6 \pm 1.04$ & $16.2 \pm 0.57$ & $18.5 \pm 0.5$ \\
\hline
\end{tabular}

$*$ Mean \pm Standard deviation.

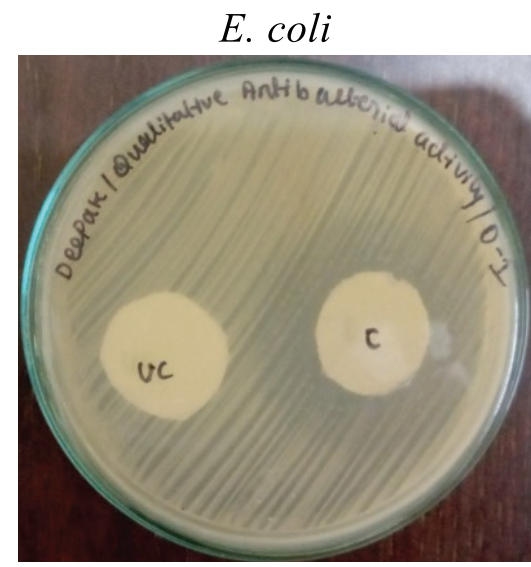

Table 3. Qualitative antibacterial activity of AMPs + PVA-coated materials

\begin{tabular}{clc}
\hline \multirow{2}{*}{ S. No } & Test organism & Zone of inhibition $(\mathbf{m m})^{$\cline { 2 - 2 }$}$ \\
\cline { 3 - 3 } 1 & E. coli & AMPs + PVA coated $^{\mathrm{a}}$ \\
\hline 2 & S. aureus & $33.1 \pm 2.31$ \\
3 & K. pneumoniae & $32.3 \pm 1.04$ \\
4 & P. aeruginosa & $27.6 \pm 1.04$ \\
5 & A. baumannii & $30.1 \pm 2.51$ \\
\hline
\end{tabular}

${ }^{a}$ Mean \pm Standard deviation

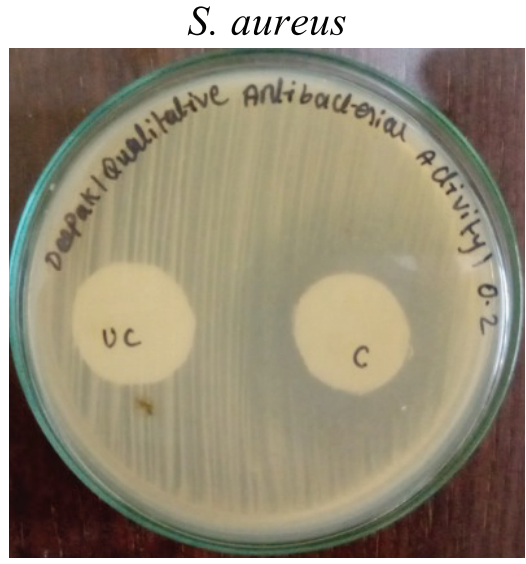

Figure 3. Qualitative antibacterial activity of the AMPs + PVA-coated wound dressing mesh samples. $\mathrm{UC}=$ Uncoated (bare wound dressing mesh without PVA and AMPs) $\mathrm{C}=\mathrm{AMPs}+\mathrm{PVA}$-coated wound dressing mesh.

$1.6 \mathrm{~mm}$ against their respective $2 \mathrm{X}, 3 \mathrm{X}$, and $4 \mathrm{X}$ concentrates. $S$. epidermidis showed $11.6 \pm 1.04,16.2 \pm 0.57$, and $18.5 \pm 0.5 \mathrm{~mm}$ of inhibitory zones against the respective concentrates.

\section{Qualitative antibacterial activity of AMPs + PVA-coated materials}

AMPs and their concentrations selected earlier were coated onto wound dressing meshes and its antibacterial potential was evaluated under in vitro conditions. Antibacterial activity was determined using the diffusing ability of the coated wound dressing samples and control samples on MHA plates. During the analysis, the zone of inhibition measured in millimeters for coated and uncoated mesh (bare material without PVA and AMPs) was calculated to obtain the mean value (tested in triplicates). In Table 3 and Figure 3, the difference in size of inhibitory zones for coated mesh and control was presented.

The most significant test organisms E. coli and S. aureus were observed to be highly susceptible to the AMPs + PVA combination. E. coli and $S$. aureus exhibited inhibitory zones of $33.1 \pm 2.31 \mathrm{~mm}$ and $32.3 \pm 1.04 \mathrm{~mm}$ for the coated mesh samples, respectively. $K$. pneumoniae and $P$. aeruginosa exhibited the inhibitory zones of $27.6 \pm 1.04 \mathrm{~mm}$ and $30.1 \pm 2.51 \mathrm{~mm}$ against 
their respective coated samples. A. baumannii showed significantly $31.1 \pm 1.73 \mathrm{~mm}$ of inhibitory zones for the test samples.

From the obtained results it was noted that AMPs + PVA-coated wound dressing mesh samples exhibited significant antibacterial activity against all the test organisms. It was mainly due to the antibacterial potential of the carrier (PVA) natively. The obtained results revealed that the carriers increase the therapeutic effect of AMPs resulting in greater inhibitory zones against the test organisms. The mode of action of PVA on the bacterial cell wall that emphasizes its antibacterial activity was reported. PVA has the ability to penetrate the surface of the bacterial pyramidal wall so that they can disassemble and prevent bacteria from forming a biofilm consequently (Salman et al., 2014). Santiago-Morales et al. (2016) also explained the mode of action of PVA. The antibacterial activity of PVA was attributed to the chelation of the divalent cations and destabilization of the peptidoglycan layer of bacteria.

The antibacterial activity aided by PVA, with antibacterial drugs surveyed from the literature, was found to be supportive with the results of the present research. Salman et al. (2014) studied the antibacterial and anti-adhesive effect of PVA and PVA/Biosufactant mixture against pathogenic bacteria. The study revealed that there is high overlap between PVA and biosurfactant molecules which improve antibacterial activity than each one separately. In another study, Santiago-Morales et al. (2016) studied the antimicrobial activity of poly(vinyl alcohol) and poly(acrylic acid) electrospun nanofibers. The antimicrobial effect was assessed using strains of $E$. coli and $S$. aureus. Membranes containing $>35 \mathrm{wt} \%$ PVA displayed significant antibacterial activity, which was particularly high for the gram-positive $S$. aureus.

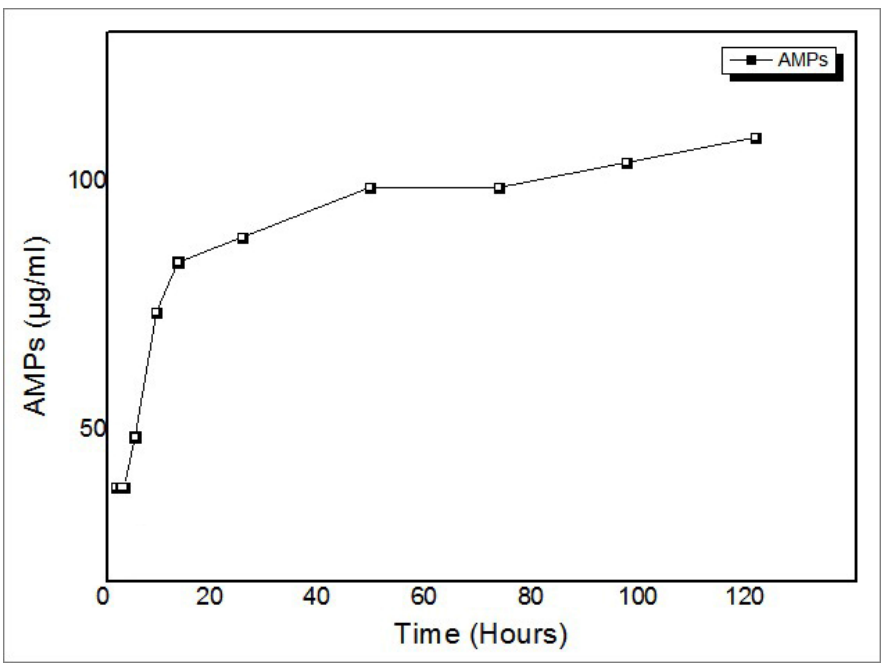

Figure 4. Determining the release concentration of AMPs from coated materials. Graph output software: ORIGIN PRO 8.6 version.

\section{Determining the release concentration of AMPs from coated materials}

An in-vitro release study was conducted on developed AMPS + PVA-coated wound dressing mesh samples. The release concentration of AMPs in PBS at a specific temperature was determined. The release study was conducted for 120 hours in PBS at $37^{\circ} \mathrm{C}$. Mesh materials coated with AMPs when released from crystalline AMPS + PVA mixture indicated that the rate of drug release was exponentially related to the release time (Fig. 4). The lag phase exhibited initial burst effect from 0.5 to 4 hours (35, $35,35$, and $45 \mu \mathrm{g})$. Followed this lag phase, an increase in drug concentration was observed from 8 to 24 hours $(70,80$, and 85 $\mu \mathrm{g})$. In PBS at $\mathrm{pH} 7.0$, the hydrophilic polymer, PVA undergoes degradation during the log phase. Due to the rate of polymer degradation, the release of drugs was facilitated at higher rate than the initial burst level concentration. During this phase, the release concentration of AMPs remained almost constant $(100,100,110$, and $120 \mu \mathrm{g}$ ) from 48 to 120 hours, indicating the sustained rate of drugs from the coated mesh samples.

The rate of degradation of the PVA in PBS will influence the wound healing process at the critical wound site. PVA is a hydrophilic polymer and its solubility in water increases greatly as the degree of the acetate group hydrolysis increases. The use of PVA as a polymer in the present research offers the possibility to place hydrophilic drugs on the surfaces of hydrophobic wound dressing materials. This slow-release drug delivery system containing AMP + PVA mixtures from mesh surface could be a promising process for vascular wound healing in the later stages (Puranik et al., 2013). Hence, PVA can be used for releasing the drugs from biological and medical materials in a controlled way (Kenawy et al., 2007).

\section{Assessing the biocompatibility of AMPs + PVA-coated materials}

The biocompatibility of the wound dressing mesh is to be ensured for the patients' safety before being used at the targeted wound site. Therefore, the biocompatibility of AMPs + PVAcoated mesh samples was evaluated by cytotoxicity assay (MTT assay). This method has an advantage over in vivo animal studies and other in vitro cell line studies. This method is very sensitive and accurate to prove the biocompatibility of any surface modified medical products. AMPs + PVA-coated mesh samples did not reduce the cell viability and cell count of fibroblast cells during the study period of up to 24 hours. The cell morphology, viability, and numbers were compared with control samples simultaneously. The results are described in Table 4 separately for each selected concentration of AMPs + PVA with the support of table values and graphical representations attributing for cell cytotoxicity and cell viability.

Table 4. Biocompatibility of the coated materials - cell viability test.

\begin{tabular}{ccccc}
\hline \multirow{2}{*}{ S. No. } & \multicolumn{3}{c}{ Fibroblast cell lines - MTT Assay } & Cytotoxic reactivity (Biocompatibility) \\
\cline { 2 - 5 } & Concentration $(\boldsymbol{\mu g})$ & ${ }^{a}$ Cytotoxicity (\%) & ${ }^{\text {a Cell viability (\%) }}$ & No cytotoxicity \\
\hline 1 & 5 & $9.3 \pm 1.04$ & $90.6 \pm 0.57$ & No cytotoxicity \\
2 & 15 & $10.6 \pm 1.08$ & $90.6 \pm 0.57$ & No cytotoxicity \\
3 & 25 & $3.6 \pm 2.51$ & $96.6 \pm 1.04$ & No cytotoxicity \\
\hline
\end{tabular}

aMean \pm Standard deviation. 


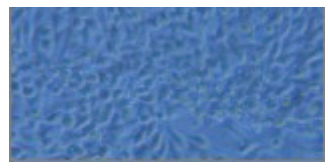

Control

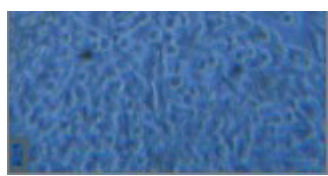

$15 \mu \mathrm{g} / \mathrm{ml}$

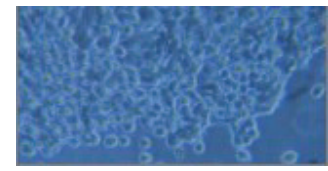

$5 \mu \mathrm{g} / \mathrm{ml}$

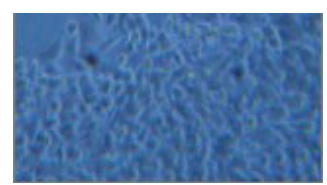

$25 \mu \mathrm{g} / \mathrm{ml}$
Figure 5. Biocompatibility of AMPs + PVA-coated wound dressing mesh. (Phase contrast microscopic analysis of $\mathrm{L}_{929}$ fibroblast cell line morphology.)

The mesh coated with selected concentrations $(5,15$, and $25 \mu \mathrm{g} / \mathrm{ml}$ ) of metabolites did not express any cytotoxic effects (Fig. 5). In support of this, an increase in cell viability with no significant difference in the morphology of the $\mathrm{L}_{929}$ fibroblast cells was evident after 24 hours of cell culturing in the cell culture media when compared to control. The results revealed that the selected concentrations did not inhibit the growth of cells; thus indicating the biocompatibility of the AMP + PVA-coated wound dressing materials.

\section{CONCLUSION}

In the present study, AMPs were extracted from marine C. malabaricus (paarai) fish. The AMPs were partially purified by ammonium sulfate precipitation and the fractional concentrations were subjected to antibacterial analysis. Based on the antibacterial potential of the extracted AMP fractions, the concentrations showing maximum inhibitory zones were selected for developing wound dressing materials. AMPs are mixed with PVA (carrier molecule) and coated on non-absorbent wound dressing materials. The most significant test organisms $E$. coli and $S$. aureus were observed to be highly susceptible to the AMPs + PVA combination. E. coli and $S$. aureus exhibited inhibitory zones of $33.1 \pm 2.31 \mathrm{~mm}$ and $32.3 \pm 1.04 \mathrm{~mm}$ for the coated mesh samples, respectively. As the antibacterial activity, drug-releasing behavior, and biocompatible properties of the coated materials showed promising results, novel tissue-engineered wound dressing materials or skin replacement equivalent are to be developed and studied as a future perspective.

\section{CONFLICT OF INTEREST}

The authors declared that they have no conflicts of interest.

\section{FUNDING}

\section{None.}

\section{REFERENCES}

Boateng JS, Mathews KH, Stevens HN, Eccleston GM. Wound healing dressings and drug delivery systems: a review. J Pharm Sci, 2008; 97(8):2892-923.

Budman DR, Calabro A, Rosen L, Lesser M. Identification of unique synergistic drug combinations associated with downexpression of survivin in a preclinical breast cancer model system. Anticancer Drugs, 2012; 23(3):272-9.
Elbadawy AK, El-Refaie SK, Chen X. A review on polymeric hydrogel membranes for wound dressing applications: PVA-based hydrogel dressings. J Adv Res, 2017; 8(3):217-33.

El-Rehewy MK, El-Feky MA, Hassan MA, Abolella HA, Abolyosr A, El-Baky RM, Gad GF. In vitro efficacy of ureteral catheters impregnated with ciprofloxacin, $\mathrm{N}$-acetylcysteine and their combinations on microbial adherence. Clin Med Urol, 2009; 3:1-8.

Elshafei A, El-Zanfaly HT. Application of antimicrobials in the development of textiles. Asian J Appl Sci, 2011; 4:585-95.

Flores A, Kingsley A. Topical antimicrobial dressings: an overview. Wound Essent, 2007; 2:182-5.

Gollwitzer H, Ibrahim K, Meyer H, Mittelmeier WR, Busch, $\mathrm{R}$, Stemberger A. Antibacterial poly (D, L-lactic acid) coating of medical implants using a biodegradable drug delivery technology. J Antimicrob Chemother, 2003; 51:585-91.

Kenawy ER, Worley SD, Broughton R. The chemistry and applications of antimicrobial polymers: a state-of-the-art review. Biomacromolecules, 2007; 8:135-9.

Mashru RC, Saikumar SV. Development and validation of spectroscopic methods for simultaneous estimation and dissolution of ofloxacin and Ornidazole in tablet dosage forms, Eclet Quim, 2010; 35(3):123-32.

Michel LL, Kamila BS, Fabricio FC. Molecular farming of antimicrobial peptides: available platforms and strategies for improving protein biosynthesis using modified virus vectors. An Acad Bras Cienc, 2019; 91(1):125-31.

Nieto Lozano JC, Meyer JN, Sletten K, Pelaz C, Nes, IF. Purification and amino acid sequence of a bacteriocin produced by Pediococcus acidilactici. J Gen Microbiol, 1992; 138:1985-90.

Puranik AS, Dawson ER, Peppas NA. Recent advances in drug eluting stents. Int J Pharm, 2013; 44(1):665-79.

Rameshkumar G, Ravichandran S, Kaliyavarathan, Ajithkumar TT. Antimicrobial peptide from crab, Thalamita crenata. World J Fish Mar Sci, 2009; 1:74-9.

Sabine AE, Martin P, Tomic-Canic M. Wound repair and regeneration: mechanisms, signaling, and translation. Sci Transl Med, 2014; $3: 62-5$.

Salman JAS, Al Kadhemy MFH, Jaleel MS, Abdal AK. Effect of PVA, PVA/biosurfactant on some pathogenic bacteria in glass and plastic plates. Int J Curr Microbiol Appl Sci, 2014; 3(10):301-9.

Santiago-Morales J, Amariei G, Leton P, Rosal R. Antimicrobial activity of poly(vinyl alcohol)-poly(acrylic acid) electrospun nanofibers. Colloids Surf B Biointerfaces, 2016; 146:144-51.

Sasmita M, Ankita A, Abhijit M. Antibacterial activity of antimicrobial peptide (AMP) grafted polystyrene surface. Adv Polym Sci Technol, 2018; 2:39-46.

White RJ. The use of topical antimicrobials in wound bioburden control. Brazilian Journal of Community Nursing, 2002; 7:20-6.

How to cite this article:

Jose DT, Uma C, Sivagurunathan P, Aswini B, Dinesh MD. Extraction and antibacterial evaluation of marine AMPs against diabetic wound pathogens. J Appl Pharm Sci, 2020; 10(11):087-092. 